\title{
Creating a successful facility for large-scale extraction of DNA, an example from the Swedish Biobank initiative BBMRI.se
}

\section{James Thompson}

Karolinska Institutet Biobank, Stockholm, Sweden

Thompson J (2015) EMBnet.journal 21(Suppl A), e830. http://dx.dọ.org/10.14806/ej.21. A. 830

Driven by the needs of several large popula- have extracted DNA from over 100000 individution cohorts, the Biobanking and BioMolecular als. Our early experience clearly demonstrated Resource Infrastructure of Sweden (BBMRI.se) has the potential of the new systems in speed and established, and now operates, a large-scale cost. But we also experienced several difficult facility for the rapid purification of high quality challenges with the approach. Genotyping and DNA from human whole blood at the KI Biobank. NGS platforms are very sensitive to DNA qualThe facility has a throughput of up to 1000 ity parameters. After gaining significant process whole blood samples per day on two parallel improvements we now see study designs that automated systems. Automation and modern use genotyping and NGS delivering results that extraction chemistry has given many benefits, promise to transform healthcare.

and since starting the operation in May 2011, we 